\title{
El Discurso de la Ciencia y la Tecnología en la Prensa Escrita Chilena: Aproximación al Corpus DICIPE-2004 (*)
}

(O Discurso da Ciência e da Tecnologia da Imprensa Escrita Chilena:

Aproximação ao Corpus DICIPE-2004)

(The Discourse of Science and Technology in the Chilean Press: an Approximation to the DICIPE-2004 Corpus)

Giovanni PARODI

Silvana FerRARI

(Pontificia Universidad Católica de Valparaíso - Chile)

RESUMO: A comunicação na ciência e na tecnologia (CET) assumiu grande relevância nos últimos anos, inicialmente através de artigos e posteriormente através dos meios de comunicação de massa. Neste contexto, os objetivos deste artigo são: a) determinar e quantificar, em termos comparativos, o espaço que um grupo de jornais chilenos destina à divulgação de temas de CET; $b$ ) determinar os tipos de texto jornalpisticos através dos quais se divulga a $C$ E T na imprensa escrita; c) identificar os macrotemas, subtemas e disciplinas presentes no corpus. O corpus foi coletado durante três meses e é formado por 411 textos. Foram calculados e normatizados as ocorrências de textos e palavras, os tipo de textos, os macrotemas, temas e disciplinas nas quais se insere cada texto. Os achados mostram, entre outros, que a divulgação de $C \in \mathcal{E} T$ ocupa, em média, $1 \%$ do que se publica nesses jornais e que predominam textos relacionados com ciências médicas, astronomia, astrofísica e ciências da vida.

PalavRas-CHAVE: discurso especializado; divulgação da C E T; imprensa escrita; lingüística de corpus.

RESUMEN: La comunicación de la ciencia y la tecnología (CET) ba cobrado gran relevancia en los últimos años, inicialmente a través de artículos científicos y actualmente

* Investigación parcialmente financiada por el Proyecto ECOS-CONICYT No CO2HO2 y por el Proyecto FONDECYT Nº 1020786.

D.E.L.T.A., 23:2, 2007 (339-369) 
a través de los medios masivos de comunicación. En este contexto, los objetivos de este artículo son: a) determinar y cuantificar, en términos comparativos, el espacio que un grupo de cinco periódicos chilenos destinan a la divulgación de temáticas de CET; b) determinar los tipos de textos periodísticos a través de los cuales se divulga la CET en la prensa escrita; c) identificar los macrotemas, subtemas y disciplinas presentes en el corpus. El corpus fue recolectado durante tres meses y quedó formado por 411 textos. Se calculó y normalizó la ocurrencia de textos y palabras, los tipos textuales, los macrotemas, temas $y$ disciplinas en las cuales se inscribe cada texto. Los hallazgos muestran, entre otros, que la divulgación de la CET ocupa en promedio un $1 \%$ de lo que se publica en estos cinco periódicos y que, mayoritariamente, predominan textos relacionados con ciencia médicas, astronomía y astrofísica y ciencias de la vida.

PalabRAs-CLAVE: discurso especializado; divulgación de la ciencia y la tecnología; prensa escrita; lingüistica de corpus.

ABSTRACT: Science and technology dissemination discourse in Chilean newspapers: approaching the Corpus DICIPE-2004. Science and technology (CET) dissemination has become a mayor field of study during last years, initially through the investigation of scientific research articles and more recently through different mass media. Our present research objectives are: a) to determine comparatively the space a group of five Chilean newspapers devote to disseminate CET topics; b) to determine newspaper text types through which CET is disseminated in written press; c) to identify macrotopics, subtopics, and disciplines to which all texts of the corpus can be adscribed. The DICIPE Corpus was collected during three months and is formed by 411 documents. Mayor findings show that CET dissemination texts are given a mean of $1 \%$ from all texts published in the five newspapers studied and that, basically, most of these texts are related to medical sciences, astronomy, astrophysics, and what could be called "life sciences".

KEY-WORDS: specialized written discourse; science and technology dissemination; newspapers; corpus linguistics.

\section{Introducción}

La sociedad de la información y del conocimiento exige que los lectores desarrollen estrategias cognitivas que les permitan procesar selectivamente grandes cantidades de información, con una adecuada capacidad crítica y conciencia de autonomía. El dominio efectivo de procesos de comprensión de textos y de aprendizaje de contenidos específicos, construidos a partir 
de múltiples fuentes, se transforman así en una necesidad cada vez más urgente.

En este línea, no constituye una novedad apuntar que los desarrollos científicos y tecnológicos han alcanzado una tremenda eclosión durante los últimos años, mas sí resulta original constatar la creciente difusión de la cual los hechos científicos son objeto. Como se sabe, la comunicación científica entre pares se realiza mayoritariamente a través de tipos de textos más o menos restringidos e institucionalizados por la comunidad en la cual se produce y disemina este conocimiento, a saber, principalmente el artículo de investigación científica (Swales, 1990, 2004; Bhatia, 1993, 2004). Cuando se piensa en una difusión a mayor escala de este conocimiento disciplinar y se busca alcanzar una audiencia más amplia, se recurre a medios diversos y se emplean estrategias discursivas que reducen la carga informativa, haciéndola más transparente a lectores menos expertos (Moirand, 2006; Parodi, 2005). En este contexto, uno de los géneros discursivos que circula con gran agilidad y al cual es factible acceder con relativa facilidad por el denominado gran público es el periodístico de prensa escrita.

Es importante puntualizar que hoy en día en Chile no conocemos en detalle los temas de ciencia y tecnología que mayoritariamente se divulgan a través de la prensa escrita, tampoco contamos con una descripción de los principales tipos de textos que se emplean para difundir temáticas de esta índole. Es acertado señalar que tampoco se dispone de un registro de los dominios disciplinares que se publican más recurrentemente, llegado el momento de divulgar este tipo de conocimiento especializado. Por supuesto que existe aún menor información en estos aspectos en el nivel contrastivo entre países de la misma lengua o el mismo continente (por ejemplo, América Latina), ni menos de lenguas diferentes. Todo ello muestra un campo fecundo de investigación.

Ahora bien, basados en los antecedentes brevemente descritos más arriba, diseñamos un proyecto de investigación cuyo objetivo es establecer un contraste entre el tipo de divulgación y de la imagen de la ciencia y la tecnología que se difunde a través de la prensa escrita tanto chilena como francesa (Proyecto ECOS-CONICYT N ${ }^{\circ} \mathrm{C02H02}$ ). De manera preliminar y más específica, los objetivos de este artículo son: a) determinar los tipos de textos periodísticos escritos a través de los cuales se divulga la ciencia y 
la tecnología (C\&T) en cinco periódicos chilenos de circulación nacional, b) cuantificar el espacio -en términos comparativos con el tamaño del diarioque estos periódicos chilenos destinan a la divulgación de temáticas de $\mathrm{C} \& \mathrm{~T}, \mathrm{y}$ c) determinar los macrotemas y subtemas que se difunden así como las disciplinas científicas en las cuales estos se inscriben. Para cumplir estos objetivos, en la primera parte del artículo, discutimos el concepto de divulgación de la $C \& \mathrm{~T}$, su rol en la práctica discursiva periodística escrita y el modo en que este discurso se puede llegar a entender como un "cuasidiscurso pedagógico". Luego, exponemos los pasos metodológicos para la recolección del corpus y los criterios de análisis. Posteriormente, se entregan los resultados y se discuten los hallazgos. Se cierra el trabajo con algunos comentarios y reflexiones.

\section{Marco de referencia}

\subsection{Discurso de divulgación científica y tecnológica}

En términos generales, entendemos la divulgación de la ciencia y la tecnología (DC\&T) como un tipo de práctica social que consiste en la difusión de un conocimiento altamente especializado, elaborado por investigadores pertenecientes a una comunidad discursiva particular, hacia un público no experto. Esta llamada popularización, difusión o divulgación implica tanto la comunicación del proceso del trabajo científico mismo, como de sus resultados. Clásicamente se ha entendido que el propósito fundamental de este proceso de re-elaboración, traducción o reformulación de información es poner a disposición de un público más amplio un conocimiento acerca de objetos, situaciones, acontecimientos o acciones relacionados con la $\mathrm{C} \& \mathrm{~T}$, muchas veces restringido solo a círculos altamente especializados (Jeanneret, 1994; Alcíbar, 2004). Respecto de los mecanismos más institucionalizados en esta difusión, De Semir (2000) revela que existen variados modos de aproximarse al proceso de divulgación y diversos actores dentro del aparato periodístico institucionalizado, los cuales no siempre cuentan con un perfil profesional definido: un periodista científico con formación especializada para abordar y re-elaborar contenidos determinados. Este asunto - mucha veces- provoca tensiones al interior de estas instituciones, tanto en el proceso de definir un camino para seleccionar temáticas y procedimientos de re-elaboración de un conocimiento, como para decidir quién es el más adecuado para esta labor. 
Por su parte, Pacheco (2003) propone que la DC\&T se constituye a través de acciones de comunicación y educación no formal sobre hechos científicos. De manera similar, Calvo (1997) opina que la DC\&T se articula como un proceso social que se proyecta a partir de la relación entre organizaciones formales (editoriales, instituciones) y la colectividad (públicos, receptores) por medio de diversos canales de difusión, asegurando la transmisión de informaciones de naturaleza científica y tecnológica, en función de intereses y expectativas (universos culturales e ideológicos particulares). Este tipo de interacción pasa por una serie de decisiones que deben ser asumidas por estamentos pertenecientes a cada institución que difunde el conocimiento especializado.

Cabe destacar que en las definiciones anteriores, el término "divulgación" se utiliza en su acepción más común, cuyos sinónimos son difundir, generalizar, propagar o hacer llegar al vulgo cierto conocimiento, que estaba reservado a una minoría (Moliner, 1998).

El espacio tradicional de la divulgación, así entendida, ha estado constituido mayoritariamente por los medios masivos de comunicación (MMC). Este marco de acción presenta cierto tipo de consecuencias para la actividad divulgativa, por lo que Pacheco (2003: 60) plantea acertadamente que:

"La divulgación es en sí misma un acto político, porque admite en ella la representación de valores, fines y creencias sobre todo lo relacionado con lo científico; en consecuencia el divulgador es un actor de la política científica”.

Ahora bien, al igual que ha acontecido en la evolución de la ciencias del lenguaje, estimamos que es posible determinar un cambio en la mirada de los investigadores sobre el objeto de estudio de la DC\&T. No es que haya ocurrido necesariamente un cambio en los textos analizados, sino que detectamos una evolución en el énfasis del foco del investigador, del analista (del observador); no en los intereses del divulgador. El cambio de foco que se produce en la forma de aproximarse a la DC\&T permite distinguir -al menos- tres tendencias o momentos. Estos cambios de mirada o de énfasis en lo estudiado no son necesariamente etapas claramente delimitadas ni cronológicamente organizadas; por el contrario, ellas coexisten, se entremezclan y permanecen a pesar de la evolución que se observa en la mirada del analista. 
Una primera mirada, prototípicamente más clásica y estrictamente más lingüística, ha sido la cultivada, entre otros, por Jeanneret (1994), Calsamiglia (1996, 2000), Calsamiglia y van Dijk (2004), Cassany (2001), Cassany, López y Martí (2000), Ciapuscio (1997, 2000, 2001). Desde esta mirada que podemos denominar tradicional, se entiende que, en el proceso de la DC\&T, un conocimiento producido en un medio y restringido a especialistas es re-escrito (esto es, vulgarizado, popularizado) para ponerse al alcance de un publico más amplio (supuestamente lego, medianamente lego o en formación en la materia). La idea central enfatizada aquí es que se difunde un saber desde un ámbito a otro a través de ciertas técnicas y estrategias discursivas; esto quiere decir que un texto producido a través del proceso de divulgación se convierte en un texto secundario, generado a partir de una fuente primaria. Diversos trabajos han abordado la indagación de los mecanismos a través de los cuales un determinado conocimiento experto es reformulado para alcanzar un nuevo estatus en el que se estudia. En estos trabajos se estudia, por ejemplo, los ajustes léxicos, las reducciones sintácticas, las transformaciones y reformulaciones, y las elisiones de núcleos conceptuales (Ciapuscio, 2003; Alcíbar, 2004; Gallardo, 2005; Marinkovich, 2005).

Un segundo momento a distinguir es aquel en que se tiende a desenfatizar el estudio del texto mismo y se focaliza el medio en que se produce esta difusión. Así, se instala la preocupación por el mediador, es decir, adquieren mayor relevancia los participantes. En esta línea, por ejemplo, Moirand $(1999,2001)$ argumenta que -en los últimos años- se ha venido conformando un nuevo tipo de comunicación divulgativa a través de los MMC. Moirand (2003) sostiene que es al llamado "mediador", a quien se le asigna un rol mucho más participativo y comprometido en el proceso de relacionar la ciencia, con sus investigadores y con el público, que el que se otorgaba al llamado "divulgador". Por su parte, Charaudeau (2003) destaca, en el marco de lo que él denomina el contrato de comunicación mediática, que una de las finalidades de este contrato es "captar a la audiencia". Este hecho implica, por una parte, que la DC\&T tiende a producir un objeto de consumo mercantil, buscando "captar a la mayoría para sobrevivir a la competencia” (Charaudeau: 2003: 85). Por otro, apunta a un objetivo ético en que se busca "seducir para educar".

Un tercer enfoque, con propósitos diferentes a los dos anteriores, ha emergido -en parte- desde Europa y ha acuñado el nombre de divulgación 
de la ciencia y la tecnología controversial o polémica. En particular, esta aproximación, políticamente más comprometida, ha emergido a partir de trabajos recientes en DC\&T referidos a temas como la salud pública, el medio ambiente y productos alimenticios. De manera más particular, han cobrado relevancia los temas acerca de los organismos genéticamente modificados (OGM) y la manipulación genética (MG). Algunos trabajos de esta índole son los de Cook (2005), Cook, Pieri y Robbins (2004, 2006), Moirand (2003, 2006), Charaudeau (2004), Marinkovich y Ferrari (2006). Desde esta mirada, lo que se busca ya no es necesariamente el análisis de estrategias discursivas de reformulación ni el estudio del mediador que difunde los conocimientos especializados, sino que se persigue -de manera especial- despertar conciencia en los lectores del uso irrestricto de cierta tecnología a través de mecanismos discursivos específicos. También el investigador busca, entre otros, poner de relieve los peligros que existen en determinado tipo de experimentación científica, alertar respecto de desarrollos tecnológicos nefastos para los seres humanos y el medio ambiente, denunciar manejos políticos, económicos y sociales en el marco de los desarrollos en C\&T.

Como es de suponer, es muy factible que esta concepción de la DC\&T haya estado implícita en la mirada denominada como más clásica y luego en la más mediática, pero queda claro que esta opción más vanguardista persigue objetivos más activistas e ideológicamente comprometidos.

Tal como señalamos, esta distinción tripartita responde más que nada a un énfasis, a la evolución de una forma de análisis más rica e interdisciplinaria en la que no necesariamente se pierden los aportes y abordajes iniciales. Desde este último y tercer enfoque, es evidente que en el análisis de la DC\&T debe existir un compromiso democratizador que resulta altamente valioso y que busca propiciar cambios en la conciencia de los lectores y sus capacidades reflexivas. Si la DC\&T sólo es concebida como el traspaso de una información altamente especializada a otra versión más simple con fines exclusivos de informar a un público más amplio, estamos en presencia de un proceso de popularización de un producto de cultura para -todavía- una elite social y no se buscaría verdaderamente alcanzar al gran público. El objetivo realmente pedagógico es escaso o nulo ya que faltan estrategias reales de incorporación del otro, de atender a sus intereses y necesidades. No solo de difundir una información realizando un proceso de re-escritura simplicada. 
Por su parte, Pacheco (2003) también adscribe a esta función ideológica de la DC\&T y postula que ella debe ayudar a conocer los límites y posibilidades de la ciencia y la tecnología y, por consiguiente, los diversos principios -entre otros- de orden político, social, económico y ético que la condicionan. Esta visión de la DC\&T contribuirá a enriquecer este quehacer y mostrará, según Pacheco (2003), sus verdades potencialidades democratizadoras en virtud de una sociedad mejor.

Como se aprecia, la divulgación de la ciencia y de la tecnología ha dejado de ser un campo de estudio relativamente ingenuo e inocuo, en el cual supuestamente se ejercía un propósito altamente positivo y neutral como era llevar a un público más amplio un conocimiento originalmente restringido a un reducido grupo de especialistas. Sin embargo, hoy se cuestiona si efectivamente este procedimiento de recontextualización de un conocimiento altamente especializado, por medio de un conjunto de estrategias de difusión, logra mantener un grado de confiabilidad con el conocimiento fuente original. $\mathrm{O}$, por el contrario, dada la exigencia de recontextualización, una parte importante de su contenido nuclear podría resultar trastocado y no corresponder al espíritu de la fuente original.

Ahora bien, si el discurso divulgativo pretende llegar al gran público y no solo transmitir un conocimiento sino que alertarlo acerca de la necesidad de tomar conciencia de hechos controversiales acerca de $C \& T$ y de posibles manipulaciones al lector (van Dijk, 2006), el discurso divulgativo de prensa escrita -sin lugar a dudas- también se transforma en un instrumento educativo por excelencia y, por ende, ostenta rasgos pedagógicos. En lo que sigue abordamos brevemente este punto.

\subsection{Discurso especializado divulgativo de la prensa escrita: ¿discurso pedagógico?}

En virtud de lo dicho anteriormente, si el discurso periodístico escrito de divulgación cumple entre otras una función educativa, resulta altamente relevante situarlo en el marco de una teoría rica y apropiada, como es la propuesta de Basil Bernstein.

Los desarrollos de la teoría de Bernstein, cuya concepción hoy en día se asume desde un enfoque conceptual discursivo de orden superior, en el 
cual la teoría clásica de los códigos queda incluida, permiten entender que el discurso especializado en su modalidad divulgativa periodística escrita corresponde a lo que Bernstein (2000) denomina un "cuasi-discurso pedagógico". Como se sabe, el autor (Bernstein, 2000) está interesado en las relaciones que dan forma a la comunicación pedagógica y sus contextos relevantes. Este investigador distingue tres formas básicas de relaciones pedagógicas, a saber, explícitas, implícitas y tácitas. Todas ellas pueden ocurrir a través de medios formales e informales. Estas relaciones se organizan en virtud del propósito del comunicador, de la visibilidad de dichos propósitos y de la conciencia de la audiencia de aquellos propósitos en que está involucrada. De este modo, en las explícitas o implícitas se opera desde la intención de iniciar, modificar, desarrollar o cambiar un conocimiento, conducta o práctica (Bernstein, 2000). Quien se supone debe adquirir este conocimiento o conducta puede o no definir esta relación como legítima o aceptar del todo lo que se pretenda que adquiera. Por último, según Bernstein (2000), la relación tácita es aquella en donde la iniciación, modificación, desarrollo o cambio del conocimiento, conducta o práctica ocurre sin que ninguno de los miembros participante estén conscientes de ello.

Dado que en el caso de los discursos de los MMC no siempre es posible contar con un fuerte control directo (o incluso indirecto) del contexto, de las relaciones sociales y de las motivaciones de los consumidores, respecto de qué es exactamente adquirido y con qué propósito, Bernstein es muy claro en apuntar que estas cuestiones están abiertas a franco debate. Por todo lo anterior, tal como se indicó más arriba, Bernstein no es definitivo al considerar el discurso de los MMC como un discurso decididamente pedagógico, pero sí de carácter cuasi-pedagógico. Ello, dado que en muchos de los discursos especializados divulgativos de tipo periodístico sí se cumplen algunos de los aspectos básicos propuestos por Bernstein para un efectivo proceso de comunicación pedagógica en que las realizaciones del control simbólico operan en segmentos diversos.

Así, muchas veces, la producción, distribución y circulación del conocimiento está desvinculada de las necesidades y compromisos del lector o comprendedor (o incluso del hoy en día llamado consumidor). De este modo, se ha producido un quiebre entre estas dos instancias vitales del proceso de comunicación o, como diría Bernstein (2000), se ha dislocado el conocimiento del conocedor propiamente tal. En este sentido, es factible 
que se tienda a impedir que la diseminación del conocimiento se produzca en respuesta a demandas externas emanadas de los que buscan nutrirse del conocimiento. Y, dado que serían otras las instancias que decidirían qué se selecciona, recontextualiza y divulga a través de los MMC, no siempre se satisfaría o se tomaría en cuenta a los lectores o receptores de los conocimientos divulgados.

Nos ha parecido prudente destacar estas ideas como referentes, ya que ellas serán retomadas posteriormente en el análisis de datos y nos permitirán explotar más adecuadamente los hallazgos empíricos.

\section{La investigación: marco metodológico}

\subsection{Criterios de selección del corpus DICIPE-2004}

Para seleccionar los diarios que componen el corpus de Divulgación de Ciencia y Tecnología en la Prensa Escrita (DICIPE-2004), se consideraron los siguientes aspectos: zona geográfica de circulación, horario de publicación, cantidad de ejemplares que circulan y audiencia. De este modo, logramos obtener una muestra amplia y diversificada, que produjera una mirada más rica y profunda. En cuanto a la zona geográfica, los diarios seleccionados son de circulación nacional, la información que entregan no se centra en el acontecer local ni regional, sino en asuntos nacionales e internacionales que afectan a la comunidad. Al considerar el horario de publicación y la cantidad de ejemplares, se optó por los periódicos matutinos que tuviesen un alto número de ejemplares -también denominados de tiraje elevado. Al mismo tiempo, tal como se ha señalado, se privilegió que los diarios apuntaran a audiencias pertenecientes a estratos sociales diversos. Así, los cinco diarios chilenos seleccionados son: El Mercurio, La Cuarta, La Nación, La Tercera y las Últimas Noticias.

Tradicionalmente, El Mercurio se reconoce como un diario de elite, dirigido a un público con un poder adquisitivo y nivel educativo alto. Una característica importante del Mercurio es que sigue una tendencia política conservadora. Entre los diarios seleccionados, El Mercurio es el único que utiliza el formato broadsheets $43 \times 29 \mathrm{~cm}$. durante la semana y de $58 \times 32 \mathrm{~cm}$. durante el fin de semana. Este formato permite la inclusión de textos más extensos con mucha publicidad y avisos de diversa índole. Comparativamente, el Mercurio es el diario más extenso de la muestra, 
además, es el único que se divide en distintos cuerpos, habitualmente tres durante la semana y siete el fin de semana.

La Tercera y la Nación están dirigidos a un público de estrato medioalto. El tamaño de sus páginas oscila entre los 40x30 cm. Cuando sus textos son muy extensos, se publica en dos páginas. La Tercera se caracteriza por la diversidad de temas que trata, sin embargo, se percibe una tendencia hacia los intereses del grupo empresarial y económico. La Nación es de tendencia oficialista, su línea editorial apoya las líneas del gobierno de turno y su director es nombrado por el Presidente de la República.

Las Últimas Noticias y la Cuarta son considerados tabloides, ambos miden $35 \times 28 \mathrm{~cm}$. Su formato pequeño es lo suficientemente cómodo como para ser leído en situaciones diversas. Las Últimas Noticias se caracteriza por divulgar hechos relacionados con el entretenimiento y la vida privada de personajes públicos, especialmente del espectáculo. En tanto, La Cuarta, el autodenominado "diario popular", se caracteriza por utilizar un sociolecto relacionado con los estratos sociales más desprovistos económica y culturalmente.

En cuanto al período de recolección del corpus, este corresponde a los meses de marzo, abril y mayo de 2004. Así, durante 92 días se revisaron consistentemente los cinco periódicos con el objetivo de detectar y clasificar todos los textos que abordaran temáticas de divulgación científica y tecnológica.

Una vez constituido el Corpus DICIPE-2004, se procedió a su digitalización en formato de textos planos y la aplicación de etiquetas de identificación. De este modo, al contar con el corpus en formato electrónico su manipulación y estudio resultó altamente más productiva, ahorrando entre otros- tiempo y espacio de almacenamiento. Este corpus esta disponible para su consulta en el sitio El Grial (www.elgrial.cl) (Parodi, 2007) sitio Web en el que además se alojan todos los corpus que ha trabajado en el último tiempo la Escuela Lingüística de Valparaíso (www.linguistica.cl).

\subsection{Objetivos}

Tal como se adelantó en el apartado Introducción, retomamos a continuación los objetivos de esta investigación los cuales orientarán el 
análisis de los resultados. Los objetivos de este estudio en particular son tres:

- Determinar y cuantificar, en términos comparativos, el espacio que un grupo de cinco periódicos chilenos de circulación nacional destinan a la divulgación de temáticas de $C \& \mathrm{~T}$ durante un periodo de 3 meses.

Para el logro de este objetivo, se determinará la cantidad de espacio en términos de número de textos y número de palabras que existe en cada uno de los periódicos en estudio, estableciendo un índice cuantitativo de comparación en relación a la cantidad de textos de cada diario y la cantidad de palabras promedio de cada diario.

- Determinar y comparar los tipos de textos periodísticos, a través de los cuales se divulga la C\&T en la prensa escrita en estudio.

Para esto, se clasificarán y cuantificarán los tipos de textos, tanto al interior de cada diario, como entre cada uno de ellos.

- Identificar las temáticas y la disciplina que se adscriben los textos del corpus DICIPE-2004. Este objetivo, busca avanzar hacia una descripción de la imagen de la ciencia y la tecnología (objetivo general del Proyecto ECOS-CONICYT N ${ }^{\circ} \mathrm{C02H02)}$ que transmiten los diarios a través de sus textos.

\section{Resultados}

\subsection{Numero de textos y de palabras en el corpus DICIPE-2004}

Una vez que se identificaron todos aquellos textos que abordaban temáticas de C\&T y/o que mostraban un enfoque divulgativo de asuntos científicos y tecnológicos, el corpus DICIPE-2004 quedó conformado por 411 textos con un total de 205.428 palabras, distribuidos en cinco diarios matutinos de la prensa chilena. La Tabla 1 muestra un desglose de los datos numéricos con cálculos porcentuales, los que permiten una comparación adecuada. 
Parodi \& Ferrari: El Discurso de la Ciencia y la Tecnología...

Tabla 1: Constitución del corpus DICIPE-2004

\begin{tabular}{|l|l|l|l|l|}
\hline Diarios & \multicolumn{2}{|l|}{$\mathrm{N}^{\circ} \mathrm{y} \%$ de textos } & \multicolumn{2}{l|}{$\mathrm{N}^{\circ} \mathrm{y}$ de palabras } \\
\hline El Mercurio & 165 & $41 \%$ & 112.261 & $54 \%$ \\
\hline Las Últimas Noticias & 21 & $5 \%$ & 8.364 & $4 \%$ \\
\hline La Cuarta & 53 & $13 \%$ & 9.329 & $5 \%$ \\
\hline La Tercera & 117 & $28 \%$ & 38.185 & $19 \%$ \\
\hline La Nación & 55 & $13 \%$ & 37.289 & $18 \%$ \\
\hline TOTAL & 411 & $100 \%$ & 205.428 & $100 \%$ \\
\hline
\end{tabular}

En la Tabla 1, podemos ver que la cantidad de textos y palabras varía considerablemente de un diario a otro. Observamos que El Mercurio concentra el mayor número tanto de textos como de palabras, representando el $41 \%$ de textos del corpus y el $54 \%$ del total de palabras. Comparativamente, La Tercera presenta un número elevado de textos. En relación a los otros tres diarios ocupa el $28 \%$ del total de textos del corpus; no obstante esta situación, el porcentaje de palabras (19\%) es muy cercano al porcentaje de palabras que presenta La Nación (18\%), a pesar de que este último registra un número más reducido de textos (13\%).

La Cuarta y la Nación presentan un número similar de textos, cada uno respectivamente con $13 \%$ del total del corpus; no obstante este dato similar, la cantidad de palabras registrada varía considerablemente entre ellos. La Cuarta corresponde al 5\% del total, mientras que la Nación corresponde al $18 \%$.

Finalmente, Las Últimas Noticias presenta el porcentaje más reducido de textos y palabras, $5 \%$ y $4 \%$, respectivamente. Este diario se destaca por utilizar un espacio comparativamente mínimo para la DC\&T, dato que no sorprende grandemente ya que se sabe que este periódico constituye un súper ventas en el país por dedicarse a temas de espectáculo y de la denominada "prensa rosa". En otras palabras, la escasez de textos y de 
palabras de este periódico en temas relacionados con la C\&T corrobora la imagen que se tiene de él.

Esta primera mirada de los datos del corpus nos permite señalar que El Mercurio, La Tercera y La Nación son los periódicos que destinan mayor espacio a la DC\&T y que su aporte resulta sustantivo en la transmisión de temáticas de esta índole. De acuerdo a la cifras de la Tabla 1, El Mercurio concentra cerca de la mitad de textos del corpus y se empina por sobre el $50 \%$ en lo que a palabras respecta: esto quiere decir que -de los cinco periódicos estudiados- este es el que aporta mayor información a nuestro corpus y sería el que mayor dedicación haría a la DC\&T. No obstante ello, cabe preguntarse si el número de textos y de palabras encontrados en el lapso de tiempo de 3 meses en cada diario constituye un índice relevante respecto del total del espacio que provee cada uno de estos periódicos. Dicho de otro modo, nos interesa saber el grado de compromiso real de cada diario con la DC\&T en cuanto al espacio comparativo que dedica a la DC\&T dentro de sus propios limites. En este escenario, estimamos que el modo de presentar los datos entregados no representa efectivamente lo que ocurre con el espacio que se destina a la ciencia y la tecnología en la prensa nacional, y sólo nos revela una panorámica general de la forma en que se compone el corpus recolectado.

Por ello y con el fin de describir la ocurrencia efectiva y comparativa de textos de DC\&T al interior de cada periódico y del resto de los textos allí incluidos, de forma que las comparaciones sean efectivas y veraces, se realizó un cálculo estimativo tanto de la cantidad de textos totales en todo un periódico (universo de textos periodísticos), así como de la cantidad total de palabras que aparece en cada periódico durante los tres meses de recolección. Este cálculo promedio se realizó a partir de la cantidad de textos y de palabras totales que se publicaron durante los 92 días en que se recolectó el corpus. Para su correcta comparación, posteriormente se procedió a un proceso de normalización de las cifras obtenidas.

A continuación, en el Gráfico 1, se comparan los resultados, según la cantidad de textos de DC\&T, que cada uno de los cinco diarios registra durante los 92 días de recolección. 


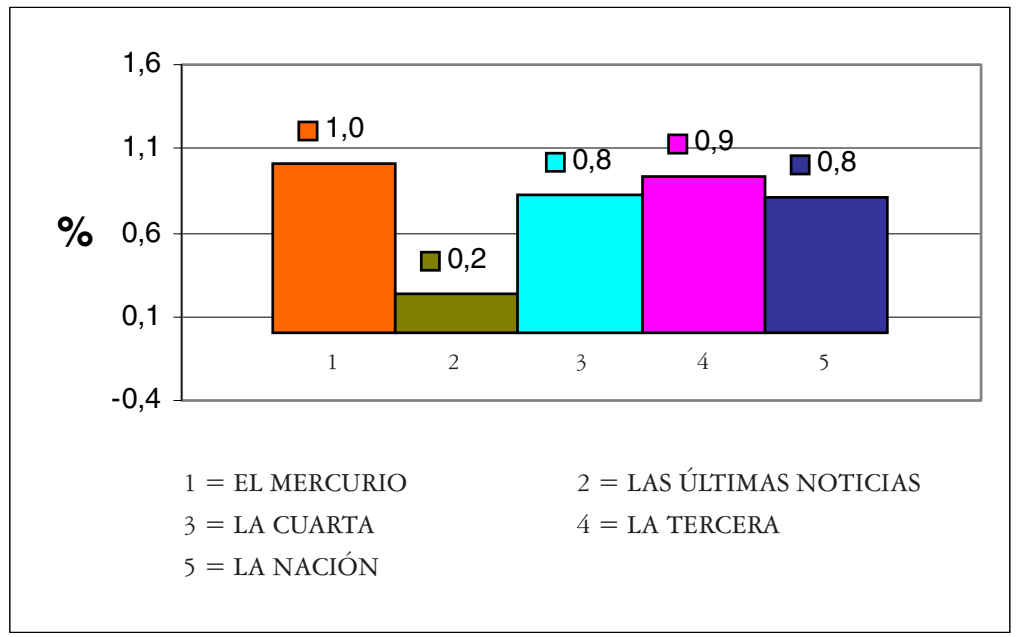

Gráfico 1: Porcentaje de textos de DC\&T por diario, calculado sobre la base del total de textos de cada diario

Según las cifras del Gráfico 1, podemos observar que, al calcular el número total de textos publicados en los cinco diarios durante los 92 días, se aprecia que las diferencias no varían en exceso entre periódicos, salvo el caso de Las Últimas Noticias $(0,2 \%)$. Se produce un cierto de equilibrio entre El Mercurio (1\%), La Tercera (0,9\%), La Cuarta (0,8\%) y La Nación $(0,8 \%)$, es decir, la cantidad de textos de DC\&T publicados en estos periódicos es relativamente homogénea, tomando como base de comparación la cantidad promedio de textos de cada diario. La excepción nuevamente es las Últimas Noticias, pues sólo el $0,2 \%$ de los textos publicados durante los tres meses de recolección tratan temas de DC\&T.

El siguiente gráfico entrega cifras comparativas, ahora por cantidad de palabras, que cada periódico destinó a DC\&T durante los 3 meses de recolección del corpus. 


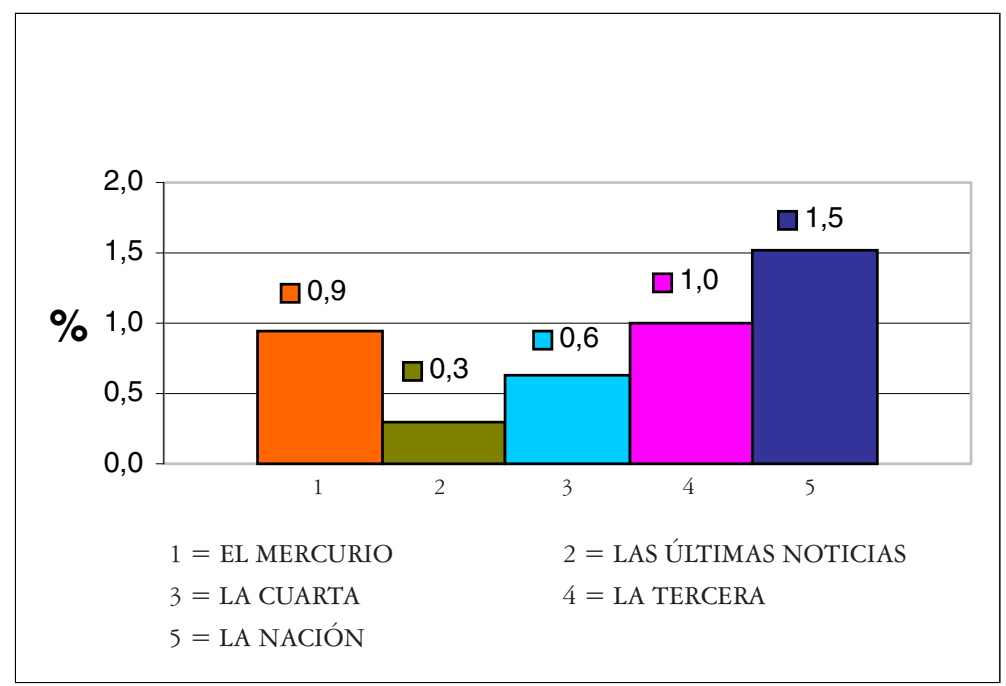

Gráfico 2: Porcentaje de palabras de C\&T por diario, calculado sobre la base del total de palabras de cada diario

Resulta interesante observar lo que ocurre en el Gráfico 2, pues aporta una nueva información que no es visible al considerar los datos presentados en la Tabla 1. En el Gráfico 2 se muestra un nuevo panorama ya no de tanta disparidad a favor de El Mercurio, en primer lugar, y La Tercera, en segunda posición (ver Gráfico 1). A partir del nuevo cálculo realizado y su normalización, observamos que los porcentajes de palabras que los diarios dedican a la DC\&T resultan heterogéneos, a pesar de la cierta tendencia regular detectada inicialmente en la cantidad de textos. Tal como se visualiza, La Nación (con el 1,5\%) es el diario que destina un mayor porcentaje de palabras a la DC\&T respecto del total de palabras que se publican, lo que lo posiciona como el medio que difunde mayor cantidad de información y que destina espacio importante para informar y educar en estos contenidos, muy por sobre El Mercurio que aparecía como liderando este asunto (ver Tabla 1). Tanto el Mercurio como La Tercera presentan porcentaje similares en cuanto al porcentaje de palabras como espacio de DC\&T (0,9 y 1 respectivamente).

Curiosamente, a pesar de que La Cuarta y La Nación presentaban un porcentaje de textos similares, difieren considerablemente en la cantidad de palabras, dicho de otro modo, puede haber muchos textos en estas 
temáticas pero son de muy escasa longitud y el espacio total destinado es más reducido que en otras fuentes periódicas en virtud de la relación del espacio total del mismo diario. Más adelante, se observará que este fenómeno tiene directa relación con los tipos textuales que los diarios utilizan para divulgar ciencia y tecnología en la prensa escrita. Finalmente, Las Últimas Noticias nuevamente presenta el porcentaje más bajo de ocurrencia y se hace congruente su análisis en virtud de lo ya señalado más arriba acerca de su carácter particular en el medio periodístico chileno.

Ahora bien, a partir de los datos entregados en los Gráficos 1 y 2, podemos concluir que el espacio que se le otorga a la divulgación de la ciencia y la tecnología en la prensa chilena no sobrepasa el $1 \%$ del total que el diario publica, ya que -en promedio- los 5 periódicos indagados dedican tan solo un $0,8 \%$ en el caso de los textos y un $0,9 \%$ en cuanto al total de palabras. No obstante, observamos algunas fuertes diferencias entre lo que los distintos diarios publican, con extremos entre La Nación y Las Últimas Noticias. Como se aprecia, estas cifras brinda una primera aproximación a nuestro objetivo y permite construir una idea inicial de la escasa preocupación que existiría en estos 5 periódicos por difundir C\&T e intentar así alcanzar un propósito pseudo-pedagógico con las audiencias implicadas. Sin lugar a dudas, se hace evidente que el compromiso institucional y periodístico es muy reducido y, a partir de estos hallazgos, se puede decir que se detecta escaso interés por parte de la prensa escrita en la divulgación de información de corte científico y tecnológico. Resulta claro que los espacios periodísticos están mayoritariamente (con cerca de un $99 \%$ ) dedicados a otros temas, los cuales habrá que indagar oportunamente.

\subsection{Los tipos textuales del corpus DICIPE-2004}

Para llevar a cabo el segundo objetivo propuesto, realizamos una revisión de las taxonomías clásicas, tanto normativas como descriptivas, que abordan la cuestión de los tipos de textos periodísticos (Calvo, 1997; Pereira, 2000; Charaudeau, 2003). Se optó por un procedimiento que reconoce en los tipos de textos rasgos convencionalmente institucionalizados y con propósitos comunicativos reconocidos (Ciapuscio, 1994, 2003; Bhatia, 1993, 2004). 
Luego de aplicar la taxonomía a los 411 textos del corpus, se comprobó que un grupo de textos no encuadraba en ninguno de los tipos convencionalmente establecidos. Se procedió a nominar y describir dicho tipo (más adelante se entregan detalles de sus rasgos, contexto de producción y sección del diario en que se publica). De este modo, Los tipos textuales se detectaron en el corpus DICIPE-2004 un total de cinco tipos textuales: Artículo Editorial, Entrevista, Noticia, Reportaje y Solicitud de Información.

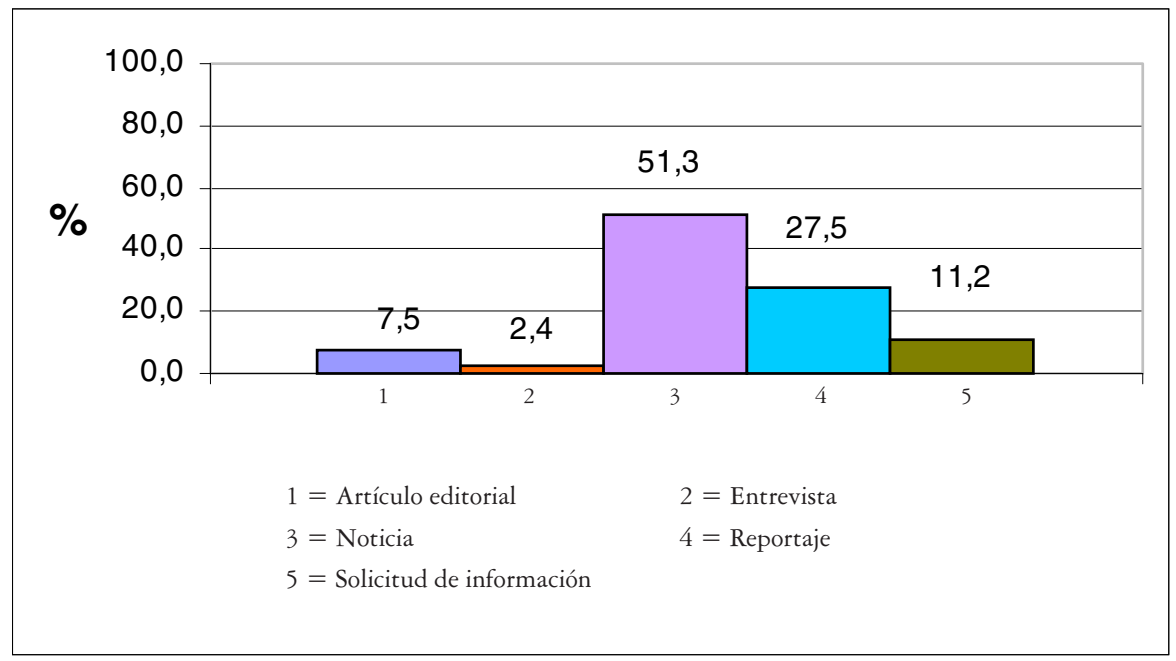

Gráfico 3: Porcentaje de los tipos textuales en el corpus DICIPE-2004

Se observa, de acuerdo a las cifras del Gráfico 3, que los tipos textuales que se utilizan en mayor medida al momento de divulgar ciencia y tecnología en el corpus DICIPE-2004 son las noticias (51,3\% textos) y los reportajes (27,5\% textos). Estos tipos de textos concentran casi el $80 \%$ de lo que se publica en prensa escrita como DC\&T, es decir, ellos se constituyen en los medios divulgativos por excelencia que el periódico elige para difundir información y conocimiento especializado. Según estos datos, las entrevistas son el tipo textual que presenta la más baja ocurrencia y tan sólo ocupa el $2,4 \%$ del corpus, seguido del artículo editorial $(7,5 \%)$ y de la solicitud de información (11,2\%). Queda claro que, en estos periódicos, las entrevistas no son el tipo de texto que se prefiere y se utiliza para difundir asuntos de C\&T. 
Por el contrario, resulta muy interesante comprobar que los periodistas, llegado el momento de difundir temáticas de C\&T, lo hacen utilizando preferentemente el tipo textual noticia. Este tipo textual les permite a los comunicadores abordar algo sensacional que ocurre tal como el descubrimiento de agua en un planeta distante, un avance bio(tecno)lógico que logrará ayudar a escoger el sexo de los hijos en el futuro cercano, o una nueva faceta de la investigación en células madre. Se detecta así, a través de este tipo textual al igual que del reportaje, una tendencia hacia lo que definimos al inicio de este artículo como la divulgación científica controversial en que, por un lado, se divulga tópicos de gran anclaje popular y, por otro, se estimula una cierta actitud reflexiva y crítica.

A continuación, en el Gráfico 4, se muestra la ocurrencia de cada uno de los tipos textuales encontrados en los cinco diarios. El porcentaje de cada tipo textual se calculó respecto del total de textos en cada diario (ver Tabla 1). Esto quiere decir que no se deben comparar estas cifras entre periódicos, sino solo entre la ocurrencia y su frecuencia al interior de cada diario.

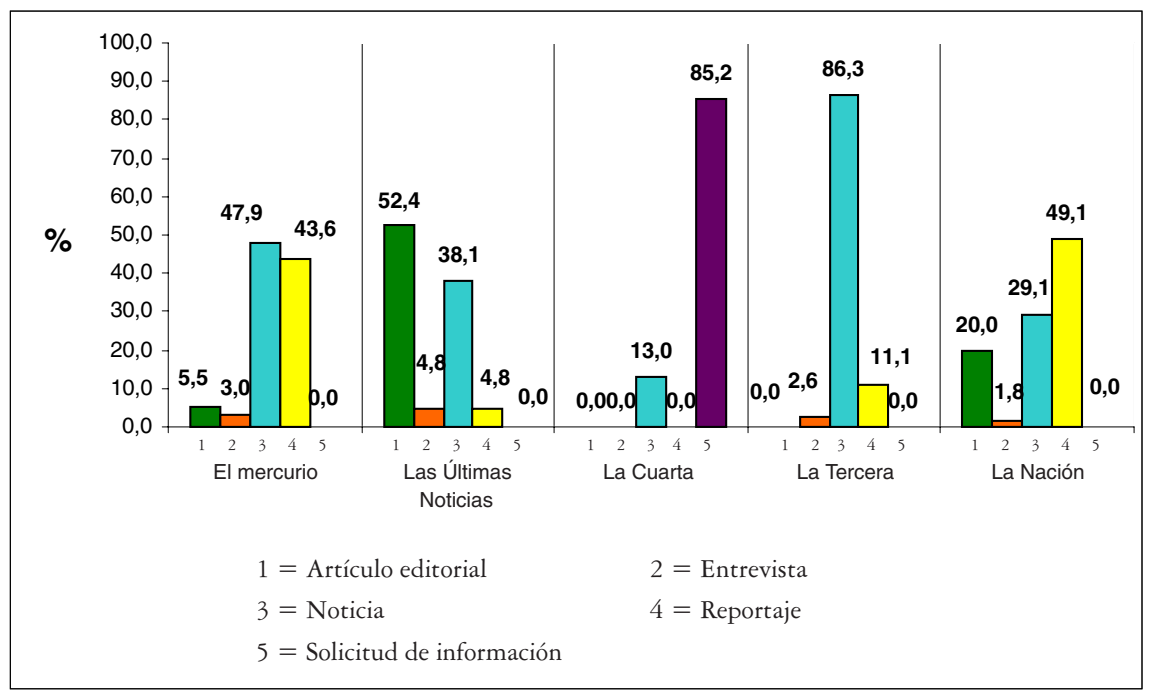

Gráfico 4: Distribución porcentual de tipos textuales por diario 
Los cinco tipos textuales se distribuyen de forma heterogénea, incluso existen diarios en que solo se encontraron algunos de los tipos detectados. Un claro ejemplo es La Cuarta. En este diario, los textos encontrados de DC\&T se clasifican en torno a solo dos tipos textuales, a saber, la noticia y la solicitud de información. Cabe destacar que este último tipo, que concentra la más alta ocurrencia $(85,2 \%)$, se encuentra exclusivamente en La Cuarta en una sección específica del diario llamada Medicina a la Carta. En esta sección, los lectores envían preguntas acerca de enfermedades y otras dolencias que son resueltas por especialistas.

Como se observa, El Mercurio utiliza mayoritariamente noticias $(47,9 \%)$ y reportajes $(43,6 \%)$ al momento de divulgar ciencia y tecnología. Lo mismo ocurre con La Nación. Sin embargo, en La Nación se observa una diferencia importante respecto del porcentaje de noticias $(29,1)$ y reportajes $(49,1)$. Estos veinte puntos de diferencia explican que La Nación sea el diario que concentra el mayor número de palabras, ya que al presentar un elevado número de reportajes, inmediatamente aumenta su número de palabras, a pesar de que su cantidad de textos es más baja que la que se registró en El Mercurio.

El artículo editorial presenta una baja ocurrencia en comparación a los otros tipos textuales. Cabe destacar que su mayor presencia se observa en Las Últimas Noticias.

\subsection{Los macrotemas y las áreas disciplinares del corpus DICIPE-2004}

En la etapa final del análisis, se aplicaron las nociones de macrotema y tema o subtema (Charaudeau, 2006) con el fin de identificar los grandes tópicos abordados en los 411 textos y alcanzar algún nivel de especificidad

por área. En la Tabla 2 se entrega una identificación en orden alfabético de los 7 macrotemas determinados y de la cantidad de textos y de porcentaje que ellos representan a partir del corpus total. 
Parodi \& Ferrari: El Discurso de la Ciencia y la Tecnología...

Tabla 2: Macrotemas en el corpus DICIPE-2004

\begin{tabular}{|l|l|l|}
\hline Macrotemas & Número de textos & Porcentaje \\
\hline Salud & 177 & $43,1 \%$ \\
\hline Medio ambiente & 125 & $30,4 \%$ \\
\hline Economía & 35 & $8,5 \%$ \\
\hline Comportamiento humano & 29 & $7,1 \%$ \\
\hline Educación & 24 & $5,8 \%$ \\
\hline Arqueología & 12 & $2,9 \%$ \\
\hline Informática & 9 & $2,2 \%$ \\
\hline Totales & 411 & $100 \%$ \\
\hline
\end{tabular}

Resulta importante observar que, de los siete macrotemas detectados, existe una alta concentración de textos en solo dos: salud $(43,1)$ y medio ambiente $(30,4)$. Según se aprecia, entre ambos macrotemas dan cuenta de más del $70 \%$ del corpus, hecho altamente relevante si se observa que la ocurrencia de los otros cinco macrotemas es relativamente baja, ya que ninguna supera el $10 \%$. Salvo que se tienda a sumarlos y se les otorgue un cierto sentido de agrupamiento en el ámbito humano y social. No obstante ello, esa cifra sobre el $20 \%$ resulta de todos modos escasa. Estos datos reflejan con claridad por donde transitan las preocupaciones e intereses de quien(es) divulga(n) y de las supuestas necesidades o motivaciones de quienes -este sujeto o institución editorial asume- recibe esta información (recordemos aquí las ideas de De Semir, 2000). Se nota con gran claridad la decisión de abordar mayoritariamente cuestiones de alta pertinencia en la vida diaria de las personas como son asuntos de medicina y enfermedades. Al mismo tiempo, estas cifras muestran una escasa atención a temáticas, por ejemplo, de tipo educativo $(5,8 \%)$. Además, muchas veces, cuando éstas son abordadas, el foco no está puesto en la popularización de ideas o contenidos acerca de difundir o cuestionar un modelo educativo o un determinado avance pedagógico, sino que estos se encuentran subordinados a cuestiones más instrumentales como una nueva prueba de selección universitaria o el cambio de personeros de gobierno asociados a las políticas educativas. ¿Será, entonces, que al público no le interesa este último tipo 
de temáticas? ¿Será que lo que se difunde no es lo más relevante a ciertas miradas, sino lo más sensacionalista? ¿Será que, como dice Bernstein (2000), se ha definitivamente dislocado el conocimiento entre los lectores y sus intereses y los difundidores del saber?

De este modo, si se espera que el discurso periodístico divulgativo de especialización en ciencia y tecnología asuma un rol como discurso pedagógico o cuasi-pedagógico, debe tenderse a una transacción de los contenidos a ser abordados, de modo de satisfacer las necesidades e intereses de las audiencias diversas implicadas en este proceso de diseminación de conocimientos. Estos resultados hacen ver la necesidad de considerar una mayor atención hacia contenidos no meramente instrumentales ni de abordarlos superficialmente, incrustándolos en el foco de la divulgación.

En sí mismo no es negativo que se atienda fuertemente a temáticas de salud y medio ambiente. Sin duda, ambos son temas muy relevantes. Lo que estas cifras muestran es que se produce un preocupante desequilibrio en la manera de administrar las temáticas que son objeto de divulgación y no se aprecia una mayor heterogeneidad que atienda muy posiblemente a necesidades e intereses más diversos de las audiencias. Por supuesto que queda pendiente una investigación acerca de los intereses efectivos de estas audiencias, ya que si ellas efectivamente solo se interesan en los temas altamente difundidos, el periodista estaría solo dando respuesta a las necesidades del medio. Asuntos ambos que escapan al foco de esta investigación y sobre los cuales, interesamente, habría que volver.

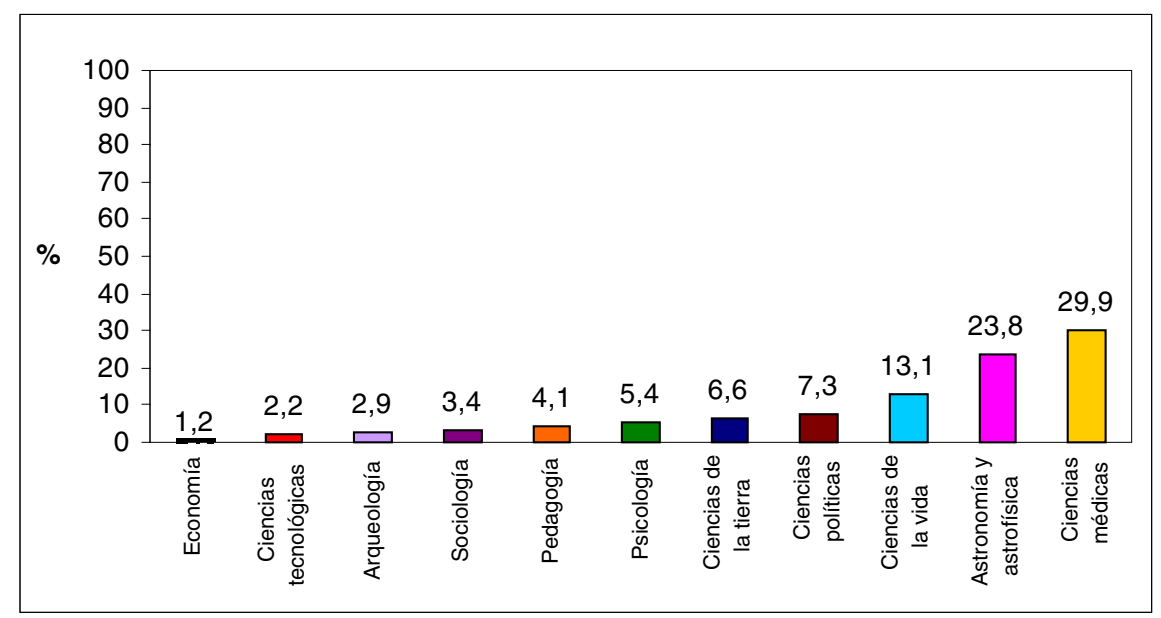

Gráfico 5: Porcentaje de textos por disciplina 
A continuación, en el Gráfico 5, se entrega la clasificación de los 411 textos del corpus en tanto a su adscripción disciplinar, con las cifras de ocurrencia normalizadas porcentualmente. Para ello se siguió la clasificación propuesta por la UNESCO (2005).

En el Gráfico 5, se observan las once disciplinas en las que se inscriben los textos que conforman el corpus DICIPE-2004. Como se aprecia, ellas presentan una dispersión heterogénea que tiende hacia una mayor concentración en tres disciplinas más o menos clásicas. Por orden de mayor a menor ocurrencia estas son: ciencias médicas, astronomía y astrofísica y ciencias de la vida. Cabe señalar en primer lugar, que esta concentración no sorprende, pues es congruente con el análisis por macrotemas en que solo dos eran mayoritarios. En su conjunto, estas tres áreas disciplinares aglutinan cerca del $70 \%$ de los textos del corpus DICIPE-2004 y tienden a incluir los dos macrotemas más productivos: salud y medio ambiente. En el polo opuesto, con la menor ocurrencia, podemos comentar tres dominios disciplinares cuya representación es muy escasa en el corpus, ya que ninguna sobrepasa el 3\%. Ellos son economía, ciencias tecnológicas y arqueología. Esta distribución porcentual resulta altamente relevante, ya que tiende a reproducir u modelo que tiende a imperar hoy en día en nuestra sociedad, en donde las ciencias humanas y sociales se encuentran desminuidas y escasamente atendidas en todo orden de cosas y las mal llamadas "ciencias duras" tienden a concentrar tanto recursos económicos, humanos como de divulgación científica, tal como estos datos reflejan certeramente. Como se aprecia, el sistema periodístico chileno, representado en estos 5 periódicos durante 92 días, ha reproducido (consciente o inconscientemente, no tenemos información al respecto) un patrón que nuestra sociedad a través de ciertos organismos institucionalizados tiende equívocamente a posicionar.

Obsérvese, comparativamente, lo escaso de la ocurrencia porcentual en la áreas de sociología, pedagogía y psicología. Así, las tres juntas alcanzan escasamente menos de un $13 \%$ promedio. Esta baja ocurrencia es inquietante y revela el sesgo que podrían estar introduciendo periodistas o entes institucionalizados en el discurso periodístico, ya sea de manera deliberada o en total inconciencia. Ahora bien, si esto se vincula con las cifras aportadas más arriba en cuanto que los diarios no dedican mas del $1 \%$ del total de sus espacios a la DC\&T, es evidente que lo que aportan a divulgar estos dominios disciplinares de ciencias sociales y humanas es casi nulo y de impacto muy relativo. Como se aprecia, no se detecta ningún 
compromiso decidido por parte de los medios por cambiar es actual estado de las cosas, sino más bien se revela una clara tendencia por reproducir las tendencias imperantes.

En resumen, las disciplinas de mayor difusión se encuentran asociados posiblemente a un mayor interés público (medicina) y a un impacto de los

Tabla 3: Distribución de los textos por tema y por disciplina

\begin{tabular}{|c|c|c|c|}
\hline TEMAS & DISCIPLINAS & $\mathrm{N}^{\circ}$ TEXTOS & $\%$ \\
\hline Hallazgos & \multirow[b]{2}{*}{ Arqueología } & 8 & 1,9 \\
\hline Patrimonio y restauración & & 4 & 1 \\
\hline Cuerpos celestes y descubrimientos & \multirow[b]{2}{*}{ Astronomía y astrofísica } & 44 & 10,7 \\
\hline Programa espacial & & 56 & 13,6 \\
\hline Cambio climático y control de fenómenos & \multirow[b]{3}{*}{ Ciencias de la tierra } & 4 & 1 \\
\hline Contaminación y energía nuclear & & 9 & 2,2 \\
\hline Extinción de especies e investigación & & 12 & 2,9 \\
\hline Organismos genéticamente modificados & \multirow[b]{3}{*}{ Ciencias de la vida } & 12 & 2,9 \\
\hline Biotecnología, clonación y fertilización artificial & & 13 & 3,2 \\
\hline Manipulación genética & & 28 & 6,8 \\
\hline Descubrimientos, enfermedades y epidemias & \multirow[b]{4}{*}{ Ciencias médicas } & 47 & 11,4 \\
\hline Ética & & 6 & 1,5 \\
\hline Fármacos y tratamientos & & 28 & 6,8 \\
\hline Nutrición, salud preventiva y síntomas & & 43 & 10,5 \\
\hline Avances tecnológicos y equipos computacionales & Ciencias tecnológicas & 9 & 2,2 \\
\hline Política y proyección económica & Economía & 5 & 1,2 \\
\hline Políticas y metodologías educacionales & Pedagogía & 17 & 4,1 \\
\hline Gestión, innovación y logros & \multirow[b]{2}{*}{ Ciencias políticas } & 27 & 6,6 \\
\hline Recursos & & 3 & 0,7 \\
\hline Aspectos de la personalidad & \multirow[b]{4}{*}{ Psicología } & 7 & 1,7 \\
\hline Desarrollo psicológico & & 5 & 1,2 \\
\hline Salud mental y terapias & & 8 & 1,9 \\
\hline Sexualidad & & 2 & 0,5 \\
\hline Comportamiento social e identidad & \multirow[b]{2}{*}{ Sociología } & 7 & 1,7 \\
\hline Organización escolar, laboral y política & & 7 & 1,7 \\
\hline TOTALES & & 411 & $100 \%$ \\
\hline
\end{tabular}


sucesos contingentes (astronomía) o a una mayor decisión editorial por fortalecer ámbitos disciplinares ya consolidados históricamente. Entre estas dos ideas centrales radica la explicación de lo que acontece a la luz de las cifras expuestas. Ahora bien, cualquiera sea el argumento que se esgrima o -incluso- uno intermedio, si se piensa en el supuesto rol de discurso pedagógico que propugnamos para la $\mathrm{DC} \& \mathrm{~T}$, ciertamente lo que aquí acontece es reduccionista y parcial.

Por último, revisamos una desagregación de los temas más específicos incluidos en los 7 macrotemas iniciales. Así, se llega ahora a un total de 25 temas más específicos y que permiten visualizar la concentración de su ocurrencia ya no globalmente por macrotemas, asunto que podría enmascarar algunos hechos puntuales. A su vez, y con el objetivo de construir un panorama final más integrador, estos temas son asociados a los once ámbitos disciplinares ya comentados más arriba a propósito del Gráfico 5 .

Los resultados de la Tabla 3 aportan nuevas evidencias que, por una parte, corroboran lo ya señalado anteriormente, pero que, por otra, ayuda a comprender más integralmente el complejo fenómeno de la DC\&T a través de la prensa escrita.

Si revisamos los temas con más alta ocurrencia porcentual, observamos que por sobre el $10 \%$ se sitúan 4 grandes temas, unidos a dos áreas disciplinares. Estos temas son, por un lado, Cuerpos celestes y descubrimientos $(10,7 \%)$ y Programa espacial $(13,6)$ del ámbito de la Astronomía y la Astrofísica; y por otro, Descubrimientos, enfermedades y epidemias $(11,4 \%)$ y Nutrición, salud preventiva y síntomas (10,5), ambos del área de las Ciencias Médicas. Estos temas más específicos así como los ámbitos disciplinares muestran la preferencia, en quien los selecciona y difunde, por asuntos de gran impacto público y que tienen alta vinculación con la vida diaria y las contingencias.

En el otro polo, encontramos los temas con menor ocurrencia o, en otras palabras, que reciben menor atención periodística. Solo comentaremos aquellos iguales o inferiores al $1 \%$. Estos son cuatro temas, vinculados a cuatro ramas disciplinares: Sexualidad $(0,5 \%)$ del ámbito de la Psicología, Recursos $(0,7 \%)$ del área de las Ciencias Políticas, Cambio climático y control de fenómenos (1\%) de las Ciencias de la Tierra y, Patrimonio y restauración (1\%) del ámbito de la Arqueología. Llama la atención encontrar el tema de la sexualidad en este grupo minoritario, el cual -por lo demás- obtiene el 
menor porcentaje de ocurrencia en total el corpus DICIPE-2004. Resulta curioso que en el mundo de hoy, con problemas tales como el SIDA, aún se tienda restringir temas como este desde una mirada de divulgación científica. Del mismo modo, dentro del listado entregado en la Tabla 3, también se consignan temas y áreas disciplinares que deberían, por muchas razones, ser de gran repercusión, pero son escasamente abordadas. Por citar solo algunas: Desarrollo psicológico (1,2\%), Ética (1,5\%), Políticas y metodologías educacionales $(4,1 \%)$.

De acuerdo a estos datos, las temáticas abordadas mayoritariamente en la divulgación de la $\mathrm{C} \& \mathrm{~T}$ durante el periodo de tres meses se abocaron básicamente a producir y reproducir un cierto patrón cultural, sesgando las posibilidades infinitas de construcción de una conciencia y una identidad ciudadana más activa, participativa y crítica. La orientación de las temáticas y sus correspondientes áreas disciplinares son una clara prueba de que el periodista, editor o institución que selecciona los ámbitos de posible difusión de conocimiento los tiende a restringir o a fortalecer aquellos clásicamente ya institucionalizados. Así, se detecta, por ejemplo, escasa atención a temas de psicología, educación, pedagogía, patrimonio cultural, recursos en su vinculación con educación, investigación, etc. También, como se argumentó más arriba, es factible que se produzca un argumento intermedio y el periodista seleccione lo que conoce que en el público tiene mayor repercusión y que representan cuestiones de mayor interés.

En este sentido, la sacralización del periodista, editor o institución como ente superior que supuestamente sabe lo que conviene al gran público para su conocimiento, información y educación es una visión equivocada que debe ser revisada. De manera más específica, un hallazgo interesante de esta investigación lo constituye lo acontecido con el periódico La Cuarta, el cual se abre a un nuevo tipo de texto (Solicitud de Información) más dialogante con la comunidad y abierto a las demandas e intereses de la misma. Habrá que ver si efectivamente se constituye como tal y no opera más bien como un recurso retórico del periodista o editor, en otras palabras, queda por indagar si son personas reales las que establecen las temáticas al solicitar información, o estas son generadas y pautadas por el equipo editorial o un periodista en particular.

Por último, es oportuno llamar la atención hacia la también excesiva sacralización de la ciencia y de la tecnología. Ello, en el sentido de que todo 
lo que produce el aparato científico y tecnológico se tiende a considerar por antomasia como positivo y en pos del bienestar del ser humano. En esto se debe ser cuidadoso y tener presente que puede existir un gran error que debe ser acotado, develado y desmitificado. Está muy claro que los propósitos últimos de algunos científicos no necesariamente ni en todos los casos constituyen en sí mismos objetivos altamente loables y se orientan positivamente en la búsqueda de la verdad. No obstante ello, es evidente que hoy en día asistimos a desarrollos tecnológicos y a aplicaciones científicas que distan mucho de proyectar el avance del ser humano hacia un enriquecimiento interior, de índole espiritual y trascendente.

\section{Comentarios finales}

Los datos aportados en esta investigación permiten concluir que, en base al análisis de corpus DICIPE-2004, la divulgación de la C\&T a través de la prensa escrita periodística se plasma, por una parte, en una variedad de cinco tipos textuales: noticias, reportajes, solicitud de información, artículo editorial y entrevista. De los cuales la noticia y el reportaje son los que representan, en promedio, cerca del $80 \%$ de lo que se publica.

Por otra parte, los análisis y cuantificaciones, revelan que las disciplinas implicadas están vinculadas mayoritariamente hacia la ciencia y la tecnología en un sentido muy clásico. Tres son las de mayor repercusión; ciencias médicas, astronomía y astrofísica y ciencias de la vida. Así, se comprueba que estos tres ámbitos disciplinares dan cuenta de cerca del $70 \%$ del total de los textos del corpus. Queda claro, entonces, que las humanidades y ciencias sociales son escaso foco de preocupación para la divulgación periodística.

Otro hallazgo de importante repercusión es que en el total de los periódicos estudiados, en promedio, se destina menos del $1 \%$ del total del espacio existente a temas de divulgación científica y tecnológica. Por lo tanto, cualquier discusión respecto a tipos de textos, temáticas abordadas y ámbitos disciplinares implicados debe ser evaluada desde este dato cuantitativo que indica el espacio real que cada periódico destina a esta cuestión. En otras palabras, mientras el compromiso institucional de estos periódicos esté tan circunscrito, podría -incluso- parecer de poco valor un estudio detallado de muchas de las cuestiones tratadas en esta investigación. 
Nosotros pensamos lo contrario, ya que son estos hallazgos los que develan la situación en sí misma.

Se hace evidente que el propósito educativo o pseudos-pedagógico de la prensa escrita se ve altamente limitado, en la medida que el espacio efectivo destinado a tareas de este tipo sea mínimo y, además, de que tienda a la reproducción de patrones muy convencionalizados, como son temáticas y ámbitos disciplinares clásicos.

En este contexto, no basta con que seamos conscientes de las posibles manipulaciones que pueden lograrse a través del manejo de estrategias discursivas y con que enseñemos a adquirir conciencia de estos propósitos, muchas veces ocultos en los textos. Es cierto que debemos ser conscientes de la manipulación que se ejerce por medio del discurso y responsablemente develar y destacar esa alternativa, pero sobre todo nuestra actitud debe ser constructivista y proactiva en virtud de una sociedad justa y democrática que se construya no solo sobre a revelación de la manipulación de los individuos sino en pro de su bienestar y libre acceso a la información. Todo ello con el fin de garantizar una vida más digna y equitativa.

Recebido em junho de 2006 Aprovado em maio de 2007

E-mails: gparodi@ucv.cl silvanamargareth@yahoo.es

\section{REFERENCIAS BibLIOGRÁFICAS}

AlCíBAR, Miguel. 2004. La divulgación mediática de la ciencia y la tecnología como recontextualización discursiva. Análisis, 31: 43-70.

BнатіA, Vijay. 1993. Analysing Genre: Language use in professional settings. London: Longman.

. 2004. Worlds of written discourse. A genre-based view. London: Continnum.

Bernstein, Basil. 2000. Pedagogy, Symbolic control and Identity. Maryland: Rowman \& Littlefield Publishers.

Calsamiglia, Helena. 1996. Apuntes sobre la divulgación científica. Un cambio de registro. Textos de didáctica de la Lengua y de la Literatura, 8: $41-52$. 
2000. Decir la ciencia: las practicas divulgativas en el punto de mira. Revista Iberoamericana de Discurso y Sociedad, 2/2: 139-146.

Calsamiglia, Helena \& Van Dijk, Teun. 2004. Popularization Discourse and Knowledge about the Genome. Discourse \& Society, 15: 369-389. Calvo, Manuel. 1997. Manual de periodismo científico. Barcelona: Bosch.

Cassany, Daniel. 2001. Análisis de la divulgación científica: Modelo teórico y estrategias divulgativas. Conferencia dictada en el XIV Congreso de la Sociedad Chilena de Lingüística. Universidad de Los Lagos, Osorno, Chile.

Cassany, Daniel; López, Carmen, \& MarTí, Jaume. 2000. Divulgación del discurso científico: la transformación de redes conceptuales. Hipótesis, modelo y estrategias. Revista Iberoamericana de Discurso y Sociedad, 2/2: 73-103.

Charaudeau, Patrick. 2003. El discurso de la información: la construcción del espejo social. Barcelona: Gedisa. . 2004. La problemática de los géneros. De la situación a la construcción textual, Revista Signos, 37/56: 23-39.

. 2006. Le discours de vulgarisation scientifique. Présupposés théoriques et cadre d'analyse. En: Giovanni Parodi. Coord. Informe Final Proyecto ECOS-CONICYT N ${ }^{\circ}$ CO2HO2. Valparaíso: Chile.

Ciapuscio, Guiomar. 1994. Tipos textuales. Buenos Aires: Eudeba. . 1997. Lingüística y divulgación de ciencia. Quark, Ciencia, Medicina, Comunicación y Cultura, 7: 19-28.

. 2000. Hacia una tipología del discurso especializado. Revista Iberoamericana de Discurso y Sociedad, 2/2: 39-70.

. 2001. Procesos y Recursos de Producción Textual en la Divulgación de Ciencia. En: Jerome Brumme. Ed. La historia de los lenguajes Iberorománicos de especialidad. La divulgación de ciencia (pp.17-42). Barcelona: Vervuert/Iberoamericana.

. 2003. Formulation and reformulation procedures in verbal interactions between experts and (semi-)lay persons. Discourse Studies, 2/5: 207-234.

Cook, Guy. 2005. Genetically Modified Language. The discourse of arguments for GM crops and food. London: Routledge.

Cook, Guy; Pieri, Elisa \& Robirns, Peter. 2006. The presentation of GM Crop Research to Non-specialists: A case Study. ESRC Research Grant. Final Report, Award number R000223725. [En línea] Disponible en: http:/ /www.regard.ac.uk/ 
Cook, Guy; Pieri, Elisa \& Robbins, Peter. 2004. The scientists think and the public feels: Expert perceptions of the discourse of GM food. Discourse E Society, 15/4: 433-449.

De Semir, Vladimir. 2000. Periodismo científico, un discurso a la deriva. Revista Iberoamericana de Discurso y Sociedad, 2/2: 9-37.

Gallardo, Susana. 2005. Los médicos recomiendan: un estudio de las notas periodísticas sobre salud. Buenos Aires: Eudeba.

Jeanneret, Yves. 1994. Ecrive la sience. Formes et enjeux de la divulgation. París: Presses Universitaires de France.

Marinkovich, Juana. 2005. Las estrategias de reformulación: el paso desde un texto-fuente a un texto de divulgación didáctica. Literatura y Lingüística, 15: 191-210.

Martin, Jim. 2004. Positive discourse analysis: Power, solidarity and change. Revista Canaria de Estudios Ingleses, 49: 179-200. . 2005. Vernacular deconstruction: Undermining spin. Conferencia Plenaria, XXXII Congreso Internacional Lingüística Sistémico Funcional, Sydney, Australia.

Matthiessen, Christian. 2005. Multilingual humanity, multilingual studies: bope or despair? Conferencia Plenaria, XXXII Congreso Internacional Lingüística Sistémico Funcional, Sydney, Australia.

Morrand, Sophie. 1999. Les indicies dialogiques de contextualisation dans la presse ordinaire. Cabiers de praxématique, 33: 145-184. . 2001. Du traitement différent de l'intertexte selon les genres convoqués dans le traitement des événements scientifique-politiques. Semen, 13: 97-117.

. 2003. Communicative and cognitive dimensions of discourse on science in the French mass media. Discourse Studies, 2/5: 175-206.

. 2006. La divulgación de la ciencia y de la técnica: ¿A nuevos objetos de estudio, nuevos modelos? Revista Signos, 39/61: 231-358.

Moliner, María. 1998. Diccionario de uso del español. Madrid: Gredos.

Pacheco, Miguel. 2003. La divulgación de la ciencia en los tiempos de la postmodernidad. Revista Ciencias, 71: 56-64.

Parodi, Giovanni. 2005. Discurso especializado e instituciones formadoras. Valparaíso: Ediciones Universitarias de Valparaíso. . 2007. El Grial: interfaz computacional para anotación e interrogación de corpus en español. En: Giovanni Parodi. Ed. Lingüística de corpus y discursos especializados. Miradas múltiples. Valparaíso: Ediciones Universitarias de Valparaíso (En prensa). 
Pereira, Ana. 2000. Análisis funcional de textos. España: Ediciones Universidad de Vigo.

Swales, John. 1990. Genre Analysis: English in academic and research settings. Cambridge: Cambridge University Press. . 2004. Research genres. Cambridge: Cambridge University Press.

UNESCO. 2005 Clasificación de las disciplinas científicas. [En línea] Disponible en: http://wzar.unizar.es/invest/unesco/

van Dijk, Teun. 2006. Discurso y manipulación: discusión teórica y algunas aplicaciones. Revista Signos, 39/60:49-74. 